\title{
Methyl Glyoxal Content of Urine of Apparently Healthy Mothers with Milk Negative to Arakawa's Reaction.
}

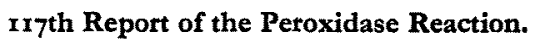

\author{
By
}

\section{Rokuro Orimo.}

(折茂六㽎)

(From the Department of Pediatrics, Faculty of Medicine, Tohoku Imperial University, Sendai.

Director: Prof. A. Sato.)

In 1931, Vogt-M $\phi$ ller ${ }^{1)}$ hypothesized that the symptoms of vitamin-B* deficiency have some connection with an intoxication of methyl glyoxal. And its poisonous character has been made known by a number of authors. 3) 3) Barrenscheen and $\mathrm{Braun}^{5}$ investigated the nature of this substance, and they described Denig è s'6) reaction with codeine phosphate and sulphuric acid, the pyrrole reaction and the reaction with 2,4-dinitrophenylhydrazine. Barrenscheen and Dreguss) devised a new colorimetric quantitative method of methyl glyoxal employing the latter reaction. By means of this method methyl glyoxal was identified from urine by many authors. ${ }^{8) 9) 10) 11) 12}$ ) From our Laboratory the toxicity of urine of

* $B_{1}$ is meant throughout the paper.

1) P. Vog t-M $\phi$ lle r, Biochem. Z., 1931, 233, 248.

2) B. Sjollem a and L. Seekles, Biochem. Z., 1926, 176, 431.

3) W. O. Kermack, C. G. Lam bie and R. H. Slater, Biochem. J., 1927, 21, 40.

4) G. Araki, Nippon Yakubutugaku Zassi, 1933, 16, 33.

5) H. K. Barrenscheen and K. Brau n, Biochem. Z., 1931, 233, 296.

6) E. Toenniessen and W. Fis cher, Z. f. phys. Chem., 1926, 161, 254.

7) H. K. Barrenseheen and M. Dreguss, Biochem. Z., 1931, 233, 305.

8) A. Geiger and A. Rosenberg, Kl. Wschr., 1933, 1258.

9) G. Popoviciu and N. Munteanu, C. r. Soc. Biol., 1934, 115, 897.

10) J. Lehman n, Skand. Arch. Phys., 1935, 71, 157.

11) A. Pi-Suner and M. Farrán, Biochem. Z., 1932, 256, 241.

12) Y. Tsukamoto and S. Tak a hashi, Zika Zassi, 1935, 418, 361. 
mothers negative to A rakawa's reaction had been reported by Suzu$\mathrm{ki}^{13){ }^{14)}} \mathrm{Suzuki}$ and Takamats $\mathrm{u}^{15)}$ and myself, ${ }^{16)}$ as I described in a preceding paper. ${ }^{16)}$ In this present paper I examined the relation between the qualitative and the quantitative methods of methyl glyoxal in urine.

\section{Method of Experiment.}

Urine was obtained from healthy mothers who visited our Dispensary. They themselves had no complaints and were "healthy." Milk samples were examined with Arakawa's reagent in each case. The cases were divided into three groups according to the intensity of Arakawa's reaction;** strongly or normally Arakawa-positive cases, weakly Arakawa-positive cases, and completely Arakawa-negative cases or cases with Arakawa's reaction $5^{\prime}(-) . * * *$ In each urine, the methyl glyoxal-like substance was examined by Fischler and Boethner's ${ }^{17)}$ method, by Barrenscheen's) reaction, by pyrrole reaction ${ }^{5)}$ and by $\mathrm{D}$ enigè s' reaction. ${ }^{6)}$ I have described these qualitative and quantitative methods in a preceding paper. ${ }^{16) 18)}$ And a case with more than 1.0 mgrm. \% was arbitrarily considered as a poisonpositive case. It will of course be a further question whether or not the poison would be methyl glyoxal itself.

In the present paper the urine content in the poison will be discussed, because, as may be understood, we were unable to obtain one whole day's urine from these " healthy" mothers.

\section{Result of Experiment.}

As above mentioned all the cases were classified into three groups according to the intensity of Arakawa's reaction. The result of the

* Arakawa-positive may be used in two different senses. One of these is: Arakawa-positive in a biochemical sense. A sample of human milk is said to have become Arakawa-positive, when it becomes blue on the addition of Arakawa's reagent. Here it means that the sample is not negative to A rakawa's reaction. The other of these two senses is: Arakawa-positive in a clinical sense. A sample of human milk is clinically Arakawa-positive only when it shows such a reaction as $H$ or $\mathrm{W}$ in one minute of the addition of $\mathrm{Arak}$ a w a's reagent. Another sample of human milk may be Arakawa-positive in the first deseribed sense, but yet clinically negative.

$5^{\prime}(-) \ldots$ A rakawa's reaction still negative after 5 minutes.

13) T. Suzuki, Tohoku J. Exp. Med., 1935, 25, 575.

14) T. Suzuki, Tohoku J. Exp. Med., 1937, 31, 280.

15) T. Suzuki and A. Takamatsu, Tohoku J. Exp. Med., 1935, 25, 588.

16) R. Orimo, Tohoku J. Exp. Méd., 1938, 33, 545.

17) F. Fis chler and R. Boethner, Ztschr. f. analyt. Chem., 1928, 74, 28.

18) R. Orim o, Tohoku J. Exp. Med., 1939, 35, 497. 
Arakawa-positive group is shown in Table 1, and that of the weakly Arakawa-positive group in Table 2, and that of the Arakawa-negative group in Table 3.

As has been shown in Table 1 there was no poison-positive case out of 30 Arakawa-positive mothers. In the weakly Arakawa-positive group, there were, as has been shown in Table 2, 6 poison-positive cases out of the 35 cases, while in the Arakawa-negative group (Cf. Table 3 ), there were 7 poison-positive cases out of the 35 cases. If now the three qualitative methods are considered, there was only one

\section{TABLE 1.}

Relation between A rakawa's reaction and qualitative and quantitative methods of methyl glyoxal-like substance in urine of lactating mothers. Arakawa-positive cases.

\begin{tabular}{|c|c|c|c|c|c|c|c|c|c|c|c|}
\hline \multirow{3}{*}{ : } & \multirow{3}{*}{ Name } & \multirow{3}{*}{ 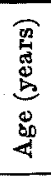 } & \multirow{3}{*}{$\begin{array}{c}\text { Diagnosis } \S \\
\text { of } \\
\text { infants }\end{array}$} & \multirow{2}{*}{\multicolumn{2}{|c|}{$\begin{array}{c}\text { Arakaw a's } \\
\text { reaction }\end{array}$}} & \multicolumn{2}{|c|}{$\begin{array}{c}\text { Denigès' } \\
\text { reaction }\end{array}$} & \multirow{3}{*}{ 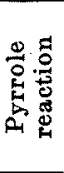 } & \multirow{3}{*}{ 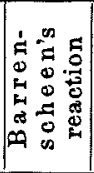 } & \multirow{3}{*}{ 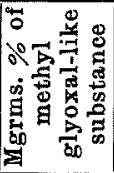 } & \multirow{3}{*}{ 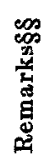 } \\
\hline & & & & & & \multirow{2}{*}{ 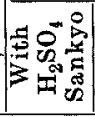 } & \multirow{2}{*}{ 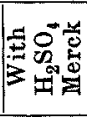 } & & & & \\
\hline & & & & Right & Left & & & & & & \\
\hline 1 & T. I. & 29 & Ang & $1(H)$ & 1 (H) & (土) & $(-)$ & $(-)$ & $(-)$ & 0 & \\
\hline 2 & T. 1. & 39 & $\mathbf{R}$ & $1(H)$ & $1(\#)$ & $(-)$ & $(-)$ & $(-)$ & $1-$ & 0.32 & \\
\hline 3 & K. S. & 30 & W & $1(\mathrm{H})$ & $1(H)$ & $(-)$ & $(-)$ & $(-)$ & $(-$ & 0.44 & $*$ \\
\hline 4 & K. S. & 28 & $W$ & $1(H)$ & $1(t+)$ & $(-)$ & $(-)$ & $(-)$ & $(-$ & 0.32 & $*$ \\
\hline 5 & T. N. & 26 & D & $1(H)$ & $1(H)$ & $(-)$ & $(-)$ & $(\dot{-})$ & $(-$ & 0 & \\
\hline 6 & F. W. & 33 & $\mathbf{E}$ & $1(H)$ & $1(H)$ & $(-)$ & $(-)$ & $(-)$ & $(-$ & 0 & \\
\hline 7 & T. H. & 45 & $\mathbf{R}$ & $1(H)$ & $1(H)$ & $(-)$ & $(-3)$ & $(-)$ & $(-$ & 0.88 & \\
\hline 8 & S. A. & 26 & Pn & $1(i)$ & $1(+t)$ & $(-)$ & $(-)$ & $(-)$ & $(-$ & 0.25 & \\
\hline 9 & Y.T. & 26 & $\mathbf{R}$ & $1(H)$ & $1(H)$ & $(-)$ & $(-)$ & $(-)$ & $(-$ & 0 & \\
\hline 10 & H. 0. & 25 & G. I & $1(t)$ & $1(+)$ & $(-)$ & $(-)$ & $(-)$ & $( \pm$ & 0.42 & \\
\hline 11 & M. S. & 27 & C. $\mathbf{H}$ & $1(H)$ & $1(+\#)$ & $(-)$ & $(-)$ & $(-)$ & $(-$ & 0.46 & \\
\hline 12 & T. A. & 26 & $\mathrm{Ma}$ & $1(H)$ & $1(t)$ & $(-)$ & $(-)$ & $(-)$ & $(-)$ & 0 & \\
\hline 13 & T. G. & 32 & H. M & $1(H)$ & $1(H)$ & $(-)$ & $(-)$ & $(-)$ & $(-$ & 0 & \\
\hline 14 & T. N. & 32 & D & $1(H)$ & $1(H)$ & $(-)$ & $(-)$ & $(-)$ & $( \pm)$ & 0.36 & \\
\hline 15 & M. M. & 26 & $\mathrm{He}$ & $1(H)$ & $1(H)$ & $(-)$ & $(-)$ & $(-)$ & (- & 0 & \\
\hline 16 & F. 0 . & 25 & W & $1(H)$ & $1(+H)$ & $(-)$ & $(-)$ & $(-)$ & (- & 0.38 & \\
\hline 17 & K. I. & 23 & At & $1(H)$ & $1(\mathbb{H})$ & $(-$ & $(-)$ & $(-)$ & $(-$ & 0.30 & \\
\hline 18 & M. A. & 44 & $\mathbf{R}$ & $1(H)$ & $1(t+)$ & $(-)$ & $(-)$ & $(-)$ & $(-$ & 0 & \\
\hline 19 & M. I. & 26 & $\mathbf{E}$ & $1(H)$ & $1(H)$ & $(-)$ & $(-)$ & $(-)$ & (- & 0.46 & * \\
\hline 20 & H. S. & 29 & G. I & $1(H)$ & $1(H)$ & (- & $(-)$ & $(-)$ & $( \pm)$ & 0.90 & \\
\hline 21 & S. M. & 29 & $\mathrm{~Tb}$ & $1(H)$ & $1(H)$ & & 83 & $(-)$ & $(-)$ & 0.56 & \\
\hline 22 & T. Y. & 36 & $\mathrm{He}$ & $1(H)$ & $1(H)$ & $(-)$ & $(-)$ & $(-)$ & $(-)$ & 0 & \\
\hline 23 & M. I. & 22 & H. 0 & $1(H)$ & $1(+t)$ & $(-)$ & $(-)$ & $(-)$ & $(-)$ & 0.40 & \\
\hline 24 & K. U. & 30 & G. I & $1(H)$ & $1(\mathrm{H})$ & -1 & $(-)$ & $(-)$ & $(-)$ & 0.38 & \\
\hline 25 & M. E. & 28 & W & $1(H)$ & $1(H)$ & $(-)$ & $(-)$ & $(-)$ & $(-)$ & 0.30 & \\
\hline 26 & R. 0 . & 21 & D & $1(H)$ & $1(H)$ & $(-)$ & $(-)$ & $(-)$ & $(-)$ & 0 & \\
\hline 27 & R. K. & 23 & At & $1(H)$ & $1(H)$ & (-) & $(-)$ & $(-)$ & $(-)$ & 0.42 & \\
\hline 28 & M. I. & 25 & E & $\mathbf{1}(H)$ & $1(H)$ & -1 & $(-)$ & $(-)$ & $(-)$ & 0.68 & * \\
\hline 29 & M. J. & 38 & Pn & $1(H)$ & $1(+t)$ & $-)$ & $(-)$ & $(-)$ & $(-)$ & 0.48 & \\
\hline 30 & K. I. & 28 & He & $1(H)$ & $1(H)$ & $(-)$ & $(-)$ & $(-)$ & $(-)$ & 0 & \\
\hline
\end{tabular}

$\S$, §§ Cf. abbreviations used in Tables 1, 2 and 3. 
Table of signs for showing different A rakawa's reaction. $\begin{array}{lllll}1^{\prime} & 2^{\prime} & 3^{\prime} & 4^{\prime} & 5^{\prime}\end{array}$

1. $1($ \#) stands for $\#$ \# H H

2. $1(+t) \quad$ \# $\#$ H

3. $2(H) \quad$ H H H H

4. $3(H)$ " $H+H$ H H

5. $4(\mathrm{H})$ " $\mathrm{H}+\mathrm{H} \mathrm{H}$

6. $5(H) \quad H+H+H$

7. $1(+) \quad+$ H H H

8. $2(+), \quad+$ H H H

9. $3(+), \quad+H+H+H$

10. $4(+)$, $\mathrm{H}+\mathrm{H}$

11. $5(+) \quad++$ H H

12. $6(+) "++\mathrm{H} H$

13. $7(+) "++\mathrm{H}+\mathrm{H}$

14. $8(+)$ " $+++H$ H

15. $9(+)$

16. $10(+)$

17. $11(+)$

18. 1 (土)

19. 2 (士)

20. $3( \pm)$

21. $4( \pm)$

22. $5( \pm)$

23. 6 (土)

24. 7 (士)
$+++H H$
$+++H H$

$++t+H$

$+++++$

$\pm+H$ H H

$\pm+H+H$

$\pm+H \mathrm{H}$

$\pm++H$ H

$\pm++H$

$\pm+++t$

$\pm t+t$ $\begin{array}{lllll}1^{\prime} & 2^{\prime} & 3^{\prime} & 4^{\prime} & 5^{\prime}\end{array}$

25. $8( \pm)$ stands for $\pm \pm+H+H$

26. 9(士) " $\pm \pm+H+H$

27. $10( \pm) \quad, \quad \pm \pm++H$

28. $11( \pm) \quad " \quad \pm \pm+++$

29. $12( \pm) \quad " \quad \pm \pm \pm++$

30. $13( \pm) \quad n \quad \pm \pm \pm++$

31. $14( \pm) \quad " \quad \pm \pm \pm t+$

32. $15( \pm) \quad, \quad \pm \pm \pm \pm \pm$

33. $1(-) \quad$ " $- \pm+H+H$

34. $2(-) \quad " \quad- \pm+H+$

35. 3(-) ", - \pm+++

36. $4(-)$ " - \pm+++

37. $5(-) \quad, \quad- \pm \pm++$

38. $6(-), \quad- \pm \pm++$

39. $7(-) \quad$ " - \pm \pm \pm+

40. $8(-) \quad, \quad- \pm \pm \pm \pm$

41. $9(-) \quad n-- \pm+H$

42. $10(-)$ " -- \pm++

43. $11(-) \quad " \quad-- \pm \pm+$

44. $12(-) \quad " \quad-- \pm \pm \pm$

45. $13(-) \quad n \quad--- \pm+$

46. $14(-) \quad " \quad--- \pm \pm$

47. $15(-) \quad " \quad---- \pm$

48. $16(-)$ " $\quad---$

Explanation to the table :-

Take, for instance, the sign : $2(H)$. This stands for A rak a wa's reaction with the course $(H) 1^{\prime}$ (H) $2^{\prime}$ (H) $3^{\prime}$ (H) $4^{\prime}$ (H) $5^{\prime}$. The sign does not express any prompt result of the reaction, so the prompt reaction of the sign: $2(H)$ may be $(-) 0^{\prime},( \pm) 0^{\prime}$, $(+) 0^{\prime}$ or even $(H) 0^{\prime}$, but this will not matter much, as the result of the reaction in one minute is the most important.

out of all these 13 poison-positive cases, in which the three reactions were all negative.

If, instead of the poison content of $1.0 \mathrm{mgrm} . \%$, a content of 0.8 mgrms. $\%$ is taken as the border line, then there were 2 " poison-positive" cases in the Arakawa-positive group, 11 cases in the weakly Arakawa-positive proup, and 12 cases in the Arakawa-negative group. Out of these 25 cases, 5 cases were negative to the three qualitative reactions. As to the average content of the poison in question, it was the largest ( $0.70 \mathrm{mgrms} . \%)$ in the completely Arakawa-negative group, the smallest $(0.29 \mathrm{mgrms} . \%)$ in the Arakawa-positive group and it was $0.58 \mathrm{mgrms} . \%$ in the weakly Arakawa-positive group. In total the average content in all the 100 cases was 0.54 mgrms. \% (Cf. Table 4).

Table 5 showed the relation between the three reactions and the "poison-positive" cases. In total Barrenscheen's reaction posi- 


\section{TABLE 2.}

Relation between Arakawa's reaction and qualitative and quantitative methods of methyl glyoxal-like substance in urine of lactating mothers. Arakawa-weakly positive cases.

\begin{tabular}{|c|c|c|c|c|c|c|c|c|c|c|c|}
\hline \multirow{3}{*}{$\begin{array}{l}0 \\
0 \\
0 \\
0 \\
0 \\
0\end{array}$} & \multirow{3}{*}{ Name } & \multirow{3}{*}{ 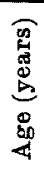 } & \multirow{3}{*}{$\begin{array}{c}\text { Diagnosis } \\
\text { of } \\
\text { infants }\end{array}$} & \multirow{2}{*}{\multicolumn{2}{|c|}{$\begin{array}{c}\text { A rakawa's } \\
\text { reaction }\end{array}$}} & \multicolumn{2}{|c|}{$\begin{array}{c}\text { Denigès' } \\
\text { reaction }\end{array}$} & \multirow{3}{*}{ 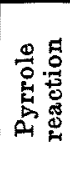 } & \multirow{3}{*}{ 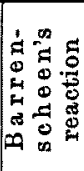 } & \multirow{3}{*}{ 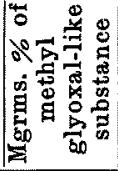 } & \multirow{3}{*}{ 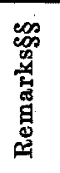 } \\
\hline & & & & & & $0^{\circ}$ & $=0^{-*} \div$ & & & & \\
\hline & & & & Right & Left & F业 & $T$ & & & & \\
\hline 1 & S. 0 . & 30 & G. I & $15( \pm)$ & $11( \pm)$ & $( \pm)$ & $(-)$ & (士) & $(-)$ & 0.78 & \\
\hline 2 & I. K. & 26 & At & $5( \pm)$ & $15(-)$ & $(-)$ & $(-)$ & (二) & $(-)$ & 1.10 & \\
\hline 3 & T. A. & 39 & $\mathrm{~L}$ ? & $7(-)$ & $7(-)$ & $(-)$ & $(-)$ & $(-)$ & $(+)$ & 0.44 & \\
\hline 4 & A. E. & 28 & Dyst & $1(+)$ & $7(-)$ & $(-)$ & $(-)$ & $(-)$ & $(-)$ & 0.35 & \\
\hline 5 & C. M. & 29 & & $14(-)$ & $11(-)$ & $( \pm)$ & $(-)$ & $(-)$ & $(-)$ & 0.88 & \\
\hline 6 & N. s. & 41 & D & $1( \pm)$ & $12(-)$ & $(-)$ & $(-)$ & $(-)$ & $(-)$ & 0 & \\
\hline 7 & K. Y. & 34 & $\mathrm{R}$ & $12(-)$ & $11( \pm)$ & $( \pm)$ & $(-)$ & $(-)$ & $(-)$ & 0.66 & " \\
\hline 8 & A. T. & 34 & D & $12(-)$ & $6( \pm)$ & $(-)$ & $(-)$ & $(+)$ & $(-)$ & 2.52 & \\
\hline 9 & T. K. & 32 & D & $5( \pm)$ & $1(H)$ & $(-)$ & $(-)$ & $(-)$ & $(-)$ & 0.24 & \\
\hline 10 & S. I. & 25 & D & $1(H)$ & $9(+)$ & $(-)$ & $(-)$ & $(-)$ & $(-$ & 0.35 & $*$ \\
\hline 11 & S. M. & 26 & D & $1(H)$ & $11(-)$ & $(-)$ & $(-)$ & $(-)$ & $( \pm$ & 0.86 & * \\
\hline 12 & I. S. & 37 & D & $6(+)$ & $11( \pm)$ & $(-)$ & $(-$ & $(-)$ & (一 & 0 & \\
\hline 13 & T. N. & 25 & $\mathrm{Pn}$ & $12(-)$ & $12(-)$ & $(-)$ & $(-)$ & $(-)$ & $( \pm)$ & 1.14 & \\
\hline 14 & T. T. & 26 & D & $6( \pm)$ & $10(-)$ & $(-)$ & $(-)$ & $(-)$ & (一) & 0 & \\
\hline 15 & K. I. & 25 & I. $\mathrm{P}$ & $15( \pm)$ & $16 c^{\prime}-$ & $(-)$ & $(-)$ & $(-)$ & $(+$ & 1.08 & $* \mathrm{~F}$ \\
\hline 16 & M. A. & 27 & $\mathrm{Br}$ & $12(-)$ & $15(-)$ & $(-)$ & & $(-)$ & $(-$ & 0 & \\
\hline 17 & T. A. & 26 & Dyst & $12(-)$ & $14(-)$ & $(-)$ & $(-)$ & $(-)$ & $(-)$ & 0 & $*$ \\
\hline 18 & T. I. & 25 & $\mathrm{Br}$ & $4( \pm)$ & $1(-)$ & $(-)$ & $(-)$ & $(-)$ & $(-)$ & 0.32 & \\
\hline 19 & T. M. & 36 & L & $5(-)$ & $16(-)$ & $( \pm)$ & $(-)$ & $(-)$ & $( \pm)$ & 0.32 & \\
\hline 20 & K. N. & 27 & Pn & $12(-)$ & $6(-)$ & $(-)$ & $(-)$ & $(-$ & $( \pm)$ & 0.89 & \\
\hline 21 & Y. K. & 27 & D & $16(-)$ & $1( \pm$ & $(-)$ & $(-)$ & $(-)$ & $(-)$ & 0.25 & \\
\hline 22 & T. K. & 27 & $\mathbf{A t}+\mathbf{E}$ & $1(-)$ & $1( \pm$ & $(-)$ & $(-$ & $(-)$ & $(-)$ & 0.36 & \\
\hline 23 & N. S. & 27 & $\mathbf{R}$ & $3(-)$ & $16(-$ & $(-)$ & $(-$ & $(-)$ & $(+)$ & 0 & \\
\hline 24 & T. M. & 24 & At & $12(-)$ & $12(-$ & $(-)$ & $(-)$ & $(-)$ & $(-)$ & 0.56 & \\
\hline 25 & H. K. & 22 & Dyst & $11(-)$ & $16(-$ & $(-)$ & $(-$ & $(-)$ & $(-)$ & 0.35 & \\
\hline 26 & T. H. & 27 & G. I $+\mathbf{E}$ & $1 \pm$ & $1( \pm$ & $(-)$ & $(-)$ & $( \pm)$ & $( \pm)$ & 0.34 & \\
\hline 27 & M. S. & 36 & $\mathrm{Ma}$ & $11(-)$ & $11(-$ & $(-)$ & $(-)$ & $(t)$ & $(+)$ & 1.53 & \\
\hline 28 & H. K. & 41 & $\mathrm{Ma}$ & $11(-)$ & $11(-$ & $( \pm)$ & $(-)$ & $(-$ & $(+)$ & 1.33 & \\
\hline 29 & Y. K. & 24 & Str & $14( \pm)$ & $12( \pm$ & $(-)$ & $(-)$ & $(-)$ & $( \pm)$ & 0 & \\
\hline 30 & U. M. & 26 & Pn & $10(-)$ & $11(-$ & $(-)$ & $(-)$ & $(-)$ & $(+)$ & 0.95 & \\
\hline 31 & S. A. & 26 & $\mathrm{Ma}$ & $6(-)$ & $3(-$ & $(-)$ & $(-)$ & $(-)$ & $(-)$ & 0 & \\
\hline 32 & Y. H. & 24 & $\mathbf{E}$ & $1(+)$ & $11+$ & $(-5)$ & $(-)$ & $(-)$ & $(-)$ & 0.60 & \\
\hline 33 & F. 0 . & 22 & He. i. s. & $7(-)$ & $7(-$ & $(-)$ & $(-)$ & $( \pm)$ & $(-)$ & 0.70 & \\
\hline 34 & Y. K. & 29 & & $8(-)$ & $81-$ & $(-)$ & $(-)$ & $(-)$ & $(-)$ & 0.88 & \\
\hline 35 & A. 0 . & 21 & $\mathrm{Ma}$ & $16(-)$ & $15( \pm$ & $(-)$ & $(-)$ & $(-)$ & $(-)$ & 0.54 & \\
\hline
\end{tabular}

$\S$, $\S$ Cf. abbreviations used in Tables 1,2 and 3.

tive $(+)$ cases were found in 13 cases, and out of these 13 there were 9 "poison-positive" cases $(69.2 \%)$ or cases with a content larger than 0.8 mgrms. $\%$. Barrenscheen's reaction positive $( \pm)$ cases were found in 15 cases, and out of these there were 8 "poison-positive" cases (53.3\%). Pyrrole reaction positive $(+$ or \pm ) was found in 16 cases, and out of these there were 8 "poison-positive" cases (50\%). Denig è s' reaction positive $( \pm)$ was found in 12 cases and out of these 
there were 5 "poison-positive" cases (41.7\%). The coincidence of Barrenscheen's reaction and pyrrole reaction (in + or \pm ) was found in 8 cases and out of these there were 6 "poison-positive" cases (75\%), and the coincidence of Barrenscheen's reaction and Denigè s' reaction (in + or \pm ) was found in 6 cases, and out of these there were 4 "poison-positive" cases (66.7\%). The coincidence of pyrrole reaction and Denigè s' reaction (in \pm or + ) was found in 3 cases and

TAble 3.

Relation between A rak awa's reaction and qualitative and quantitative methods of methyl glyoxal-like substance in urine of lactating

mothers. Arakawa-negative cases.

\begin{tabular}{|c|c|c|c|c|c|c|c|c|c|c|c|}
\hline \multirow{3}{*}{ 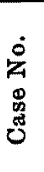 } & \multirow{3}{*}{ Name } & \multirow{3}{*}{ 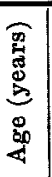 } & \multirow{3}{*}{$\begin{array}{c}\text { Diagnosis } \$ \\
\text { of } \\
\text { infants }\end{array}$} & \multirow{2}{*}{\multicolumn{2}{|c|}{$\begin{array}{l}\text { Arakawa's } \\
\text { reaction }\end{array}$}} & \multicolumn{2}{|c|}{$\begin{array}{l}\text { Denigès' } \\
\text { reaction }\end{array}$} & \multirow{3}{*}{ 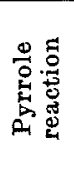 } & \multirow{3}{*}{ 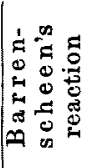 } & \multirow{3}{*}{ 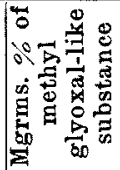 } & \multirow{3}{*}{ 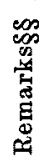 } \\
\hline & & & & & & \multirow{2}{*}{ 岳家是 } & \multirow{2}{*}{ 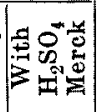 } & & & & \\
\hline & & & & Right & Left & & & & & & \\
\hline 1 & M. M. & 25 & D & $16(-)$ & $16(-)$ & $(-)$ & $(-)$ & $(+)$ & $(-)$ & 0.78 & \\
\hline 8 & A. $\mathrm{K}$. & 26 & $\mathrm{He}$ & $16(-)$ & $16(-)$ & $(-)$ & $(-)$ & $(-1)$ & $(-)$ & 0.34 & \\
\hline $\mathbf{3}$ & T. I. & 33 & De & $16(-)$ & $16(-)$ & $(-)$ & $(-)$ & $(-)$ & $(-)$ & 0.68 & \\
\hline 4 & E. $\mathbf{S}$. & 32 & I. P? & $16(-)$ & $16(-)$ & $(-)$ & $(-)$ & $(-)$ & $( \pm)$ & 0.50 & $\%$ \\
\hline 5 & T. F. & 25 & I. $\mathbf{P}$ & $15(-)$ & $15(-)$ & $(-)$ & $(-)$ & $( \pm)$ & $( \pm)$ & 0.77 & $\%$ \\
\hline 6 & T. $\mathbf{I}$ & 27 & $A t+D$ & $16(-)$ & $16(-)$ & $( \pm)$ & $(-)$ & $( \pm)$ & $(+)$ & 1.76 & \\
\hline 7 & Y. E. & 29 & I. $\mathbf{B}$ & $16(-)$ & $16(-)$ & $(-)$ & $(-)$ & $( \pm)$ & $( \pm)$ & 0.95 & $\mathrm{Be}$ \\
\hline 8 & S. W. & 33 & D & $16(-)$ & $16(-)$ & $(-)$ & $(-)$ & $(-)$ & $(-)$ & 0.46 & $*$ \\
\hline 9 & T. N. & 30 & $w+\operatorname{Imp}$ & $16(-)$ & $16(-)$ & $(-)$ & $(-)$ & $(-)$ & $(-)$ & 0 & \\
\hline 10 & M. 0 . & 38 & w & $16(-)$ & $16(-)$ & $(-)$ & $(-)$ & $( \pm)$ & $(-)$ & 0.66 & \\
\hline 11 & H. $\mathrm{O}$. & 27 & D & $14(-)$ & $16(-)$ & $(-)$ & $(-)$ & $(-)$ & $(-)$ & 0.62 & \\
\hline 12 & S. S. & 23 & D & $16(-)$ & $16(-)$ & $(-)$ & $(-)$ & $( \pm)$ & $(+)$ & 1.12 & \\
\hline 13 & H. 0. & 28 & $\mathbf{M a}+\mathbf{E}$ & $16(-)$ & $16(-)$ & $(-)$ & $(-)$ & $(-)$ & $(-3)$ & 0 & 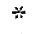 \\
\hline 14 & K. K. & 25 & $\mathrm{~L}$ & $16(-)$ & $16(-)$ & $( \pm)$ & $(-)$ & $(-)$ & $(-)$ & 0.64 & $\%$ \\
\hline 15 & M. Y. & 31 & At & $16(-)$ & $16(-)$ & $(-)$ & $(-)$ & $(-)$ & $(-)$ & 0.44 & \\
\hline 16 & F. S. & 21 & At & $16(-)$ & $16(-)$ & $( \pm)$ & $(-)$ & $(-)$ & $(+)$ & 0.68 & \\
\hline 17 & T. K. & 21 & D & $16(-)$ & $16(-)$ & $( \pm)$ & $(-)$ & $(-)$ & $i+$ & 1.22 & $\ddot{*}$ \\
\hline 18 & K. 0 . & 26 & $\mathrm{Ma}$ & $16(-)$ & $16(-)$ & $(-)$ & $(-)$ & $(-)$ & & 0.57 & \\
\hline 19 & S. 0 . & 27 & He. $\mathbf{u}$ & $16(-)$ & $15(-)$ & $(-)$ & $(-)$ & $(-)$ & $(-)$ & 0 & \\
\hline 20 & H. Y. & 24 & Dyst $+B r$ & $15(-)$ & $15(-)$ & $(-)$ & $(-)$ & $( \pm)$ & $(+)$ & 1.45 & \\
\hline 21 & K. K. & 29 & L & $16(-)$ & $16(-)$ & $(-)$ & $(-)$ & $(-)$ & $( \pm)$ & 0.86 & \\
\hline 22 & I. M. & 23 & W & $16(-)$ & $16(-)$ & $(-)$ & $(-)$ & $(-)$ & $(-)$ & 0.44 & * \\
\hline 23 & S. K. & 25 & At & $15(-)$ & $15(-)$ & $( \pm)$ & $( \pm)$ & $(+)$ & $(+)$ & 1.75 & \\
\hline 24 & Ү. H. & 31 & Ang + D & $16(-)$ & $16(-)$ & (-) & $(-)$ & $(+)$ & $(-)$ & 1.01 & \\
\hline 25 & T. N. & 26 & D & $16(-)$ & $16(-)$ & $(-)$ & $(-)$ & $(-$ & $(-)$ & 0.88 & * \\
\hline 26 & K. M. & 30 & $\mathrm{D}$ & $16(-)$ & $16(-)$ & $(-)$ & $(-)$ & $( \pm)$ & $(-)$ & 0 & * \\
\hline 27 & S. I. & 23 & Pn & $16(-)$ & $16(-)$ & $(-)$ & $(-)$ & $( \pm)$ & $(-)$ & 0.76 & \\
\hline 28 & T. S. & 26 & $\mathrm{Br}$ & $16(-)$ & $16(-)$ & $(-)$ & $(-)$ & $(-)$ & $( \pm)$ & 1.28 & \\
\hline 29 & M. 0 . & 30 & $\mathrm{D}$ & $15(-)$ & $16(-)$ & $( \pm)$ & $(-)$ & $(-$ & $(-)$ & 0.54 & \\
\hline 30 & K. S. & 42 & L? & $15(-)$ & $15(-)$ & (一) & $(-)$ & $(-)$ & $( \pm)$ & 0.85 & * \\
\hline 31 & R. F. & 23 & Pn & $15(-)$ & 160 & $(-)$ & $(-)$ & $(-$ & $(-)$ & 0.95 & \\
\hline 32 & T. M. & 28 & $\mathrm{D}$ & $15(-)$ & 15( & $(-)$ & $(-)$ & $(-)$ & $(-)$ & 0.34 & \\
\hline 33 & T. N. & 26 & Mst & $16(-)$ & $16(-)$ & $(-)$ & $(-)$ & $(-)$ & $(-)$ & 0 & \\
\hline 34 & K. I. & 32 & $\mathrm{At}+\mathrm{Hg}$ & $15(--)$ & $15(-)$ & $(-)$ & $(-)$ & $(-)$ & $(-)$ & 0.76 & \\
\hline 35 & H. T. & 38 & An+Dyst & $16(-)$ & $16(-)$ & $(-)$ & $(-)$ & $(-)$ & $(+)$ & 0.44 & \\
\hline
\end{tabular}

$\S$, $\S \S$ Cf. abbreviations used in Tables 1, 2 and 3. 
Abbreviations used in Tables 1, 2 and 3.

Be ......Mother was suffering from beriberi

*........ Mother has been taking vitamin.B

?.........Suspicion of

An ......Anemia

Ang ...Angina

At .......Atrophy

$\mathrm{Br}$......Bronchitis

C. H ...Congenital heart diseases

D ...... Dysepsia

De ......Debilitas vitae

Dyst ... Dystrophy

E ......Eczema

G. I ...Grippal infection

He ...... Healthy

He. i. s..Hernia inguinalis sinistra

He. u ...Hernia umbilicalis
Hg ......Hypogalactia

H. M ...Heine-Medin's disease

H. O ...Habitual obstipation

I. B ...Infantile beriberi

I. $\mathbf{P}$...Infantile preberiberi

Imp ...Impetigo

L ......Lues congenita

Ma ......Malnutrition

Mst ...Mastitis

Pn ......Pneumonia

R ......Rhinopharyngitis

Str...... Strophulus infantum

T ......Torticollis

$\mathrm{Tb}$......Pulmonary tuberculosis

W ......Whooping cough

out of these there were 2 "poison-positive" cases (66.7\%).

All three reaction cases were equally + or \pm in 2 cases; both cases were "poison-positive" or of a content larger 0.8 mgrms. $\%$ of the poison.

Table 6 showed the relation between the three reactions and the poison-positive cases. In total $\mathrm{B}$ arrenscheen's reaction positive $(+)$ cases were found in 13 cases, and out of these 13 , there were 8 poison-positive cases $(61.5 \%)$, or cases with a content larger than 1.0 mgrm. \%. Barrenscheen's reaction positive $( \pm)$ cases were found in 15 cases and out of these there were 2 poison-positive cases (13.3\%).

Table 4.

The distribution of poison-positive cases according to the intensity of A rak awa's reaction.

\begin{tabular}{|c|c|c|c|c|c|}
\hline \multicolumn{2}{|c|}{$\begin{array}{c}\text { Arakawa's } \\
\text { reaction }\end{array}$} & $\begin{array}{l}\text { Cases } \\
\text { in all }\end{array}$ & $\begin{array}{c}\text { Poison-positive } \\
\text { cases with a } \\
\text { larger content } \\
\text { than } 1.0 \\
\text { mgrm. } \% \\
\end{array}$ & \begin{tabular}{|c} 
"Poison-positive' \\
cases with a \\
larger content \\
than 0.8 \\
mgrms. $\%$
\end{tabular} & $\begin{array}{l}\text { Average content } \\
\text { of methyl glyoxal- } \\
\text { like substance } \\
\text { (in mgrms. \%) }\end{array}$ \\
\hline \multicolumn{2}{|c|}{ Positive group } & 30 & 0 & $2(1)$ & 0.29 \\
\hline \multirow{2}{*}{ 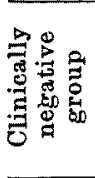 } & $\begin{array}{l}\text { Weakly } \\
\text { positive } \\
\text { group }\end{array}$ & 35 & $6(1)^{*}$ & 11 (2) & 0.58 \\
\hline & $\begin{array}{l}\text { Completely } \\
\text { negative } \\
\text { group }\end{array}$ & 35 & $7(0)$ & 12 (2) & 0.70 \\
\hline \multicolumn{2}{|r|}{ Total } & 100 & 13 (1) & $25 \quad(5)$ & 0.54 \\
\hline
\end{tabular}

* The number in parenthesis..... The number of cases in which all the three reactions were negative. 
TABLE 5.

Relation between the three qualitative reactions and the "poison-positive" cases.

\begin{tabular}{|c|c|c|c|c|c|c|c|c|c|}
\hline Ara & $\begin{array}{l}\text { k awa's } \\
\text { etion }\end{array}$ & $\begin{array}{l}\Xi \\
\Xi \\
\Xi \\
0 \\
0 \\
0 \\
0 \\
0\end{array}$ & $\begin{array}{l}\text { Cases with } \\
\text { Barren- } \\
\text { scheen's } \\
\text { reaction } \\
\text { positive }\end{array}$ & 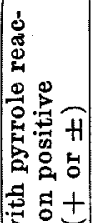 & 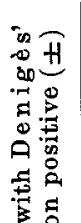 & 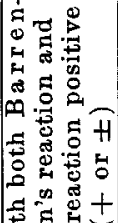 & 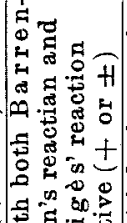 & 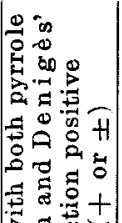 & 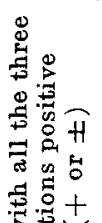 \\
\hline & & & $+\quad \pm$ & $\begin{array}{l}0 \\
0 \\
0 \\
0 \\
0 \\
0 \\
0\end{array}$ & 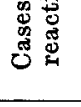 & 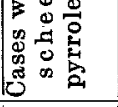 & 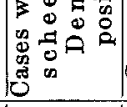 & 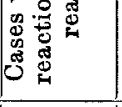 & 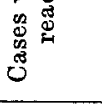 \\
\hline Positi & ve group & $\mathbf{3 0}$ & $3(1)$ & 0 & $1(0)$ & 0 & 0 & 0 & 0 \\
\hline 홀 & $\begin{array}{l}\text { Weakly } \\
\text { positive } \\
\text { group }\end{array}$ & 35 & $6(4) * 6(3)$ & $5(2)$ & $5(2)$ & $2 \quad(1)$ & $2(1)$ & $1 \quad(0)$ & 0 \\
\hline 量 & $\begin{array}{l}\text { Negative } \\
\text { group }\end{array}$ & 35 & $7(5) \quad 6(4)$ & $11(6)$ & $6(3)$ & $6 \quad(5)$ & $4 \quad(3)$ & $2 \quad(2)$ & $2 \quad(2)$ \\
\hline & & 100 & $13(9) 15(8)$ & $16(8)$ & $12(5)$ & 8 (6) & $6 \quad(4)$ & $3 \quad(2)$ & $2 \quad(2)$ \\
\hline & & & $69.2 \% 53.3 \%$ & $50 \%$ & $41.7 \%$ & $75 \%$ & $66.7 \%$ & $66.7 \%$ & $100 \%$ \\
\hline
\end{tabular}

* The number in parenthesis......The number of cases with a larger content than 0.8 mgrms. $\%$.

Pyrrole reaction positive $(+$ or \pm ) was found in 16 cases, and out of these there were 7 poison-positive cases (43.8\%). Den i gè s' reaction positive $( \pm)$ was found in 12 cases, and out of these there were 4 poison-positive cases (33.3\%). The coincidence of Barrenscheen's reaction and pyrrole reaction (in + or \pm ) was found in 8 cases, and out of these there were 5 poison-positive cases (62.5\%), and the coincidence of Barrenscheen's reaction and Denigès' reaction (in + or \pm ) was found in 6 cases, and out of these there were 4 poison-positive cases $(66.7 \%)$. The coincidence of pyrrole reaction and Denigès' reaction (in \pm or + ) was found in 3 cases, and out of these there were 2 poison-positive cases $(66.7 \%)$. All the three reaction cases were equally + or \pm in 2 cases; both cases were poison-positive or of a content larger $1.0 \mathrm{mgrm}$. \% of the poison.

\section{Preliminary Report of Infuence of Vitamin-B with or without Yakriton upon the Content of Methyl Glyoxal-like Substance in Lactating Mothers' Urine.}

By way of appendix I shall report of my clinical experiment which 
TABLE 6.

Relation between the three qualitative reactions and the poison-positive cases.

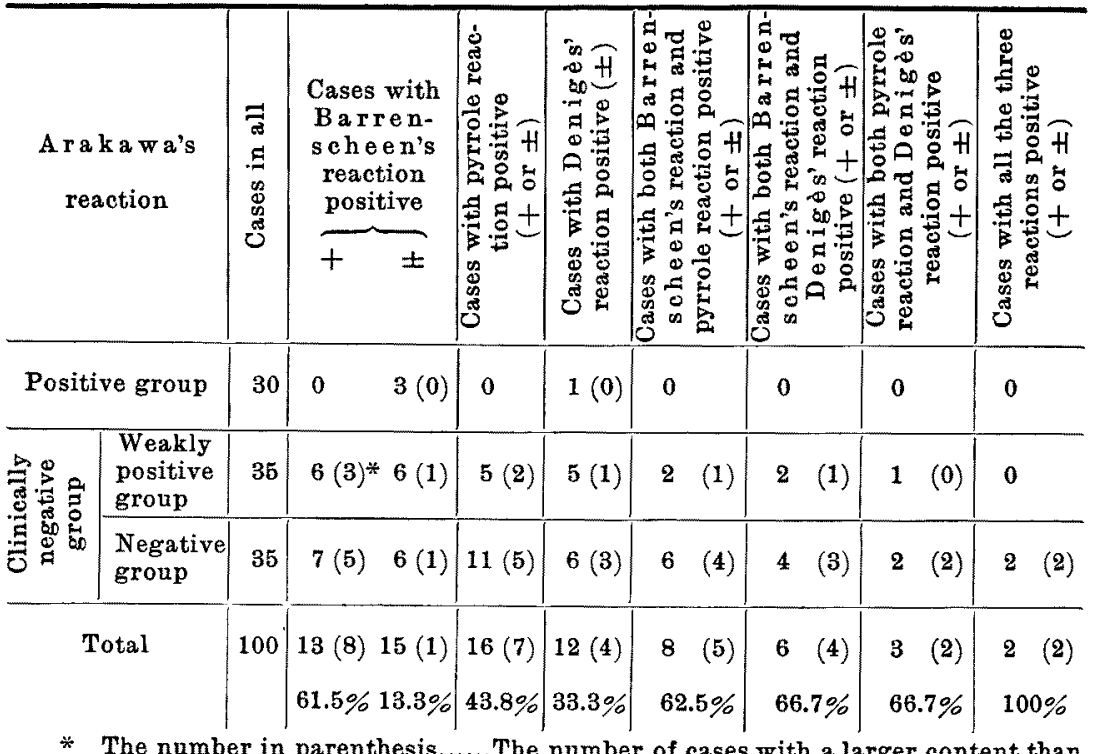
1.0 mgrm. $\%$.

tested whether vitamin-B would exert a favourable effect for decreasing the urine content in the methyl glyoxal-like substance. In all the 16 cases experimented on, there were only 4 "poison-positive" cases; in the other 12 cases the poison content was rather small, so that unprejudiced judgment may be difficult. However, there was none among the latter 12 cases that showed an increase of the content except Case 15 with no treatment. In the former 4 cases a decrease of the content was evident in three cases; in the other case (Case 1) it showed rather an increase of the content, but this was a case of beriberi and ought to have been more energetically treated. In cases 2 and 4 no vitamin-B was used, but yakriton was injected. Yakriton will be understood to have improved liver insufficiency and to have mobilized vitamin-B. The favourable influence of vitamin-B will also be seen from the three qualitative reactions (Cf. Table 7).

\section{Comment.}

From my own investigation it will be difficult to see an exact coincidence among the three qualitative methods-Barrenscheen's 


\section{TABLE 7.}

Influence of the treatment with vitamin-B (or with vitamin-B and yakriton) upon methyl glyoxal-like substance of mothers' urine and upon mothers' A rak a wa's reaction.

\begin{tabular}{|c|c|c|c|c|c|c|c|c|c|}
\hline \multirow{2}{*}{ 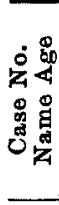 } & \multirow{2}{*}{$\begin{array}{c}\text { Diagnosis } \\
\text { of } \\
\text { infants }\end{array}$} & \multirow{2}{*}{$\begin{array}{c}\text { Date of } \\
\text { experi- } \\
\text { ment }\end{array}$} & \multicolumn{2}{|c|}{$\begin{array}{c}\text { Arakawa's } \\
\text { reaction }\end{array}$} & \multirow{2}{*}{ 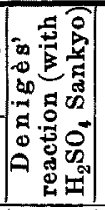 } & \multirow{2}{*}{ 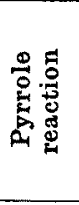 } & \multirow{2}{*}{ 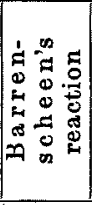 } & \multirow{2}{*}{ 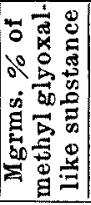 } & \multirow{2}{*}{ Remarks } \\
\hline & & & Right & Left & & & & & \\
\hline $\begin{array}{c}1 \\
\text { K.I. } \\
25\end{array}$ & Dyspepsia & $\begin{array}{l}\text { 14. } \mathrm{X} \\
\text { 19. " } \\
24 . "\end{array}$ & $\begin{array}{l}15( \pm) \\
16(-) \\
16(-)\end{array}$ & $\begin{array}{l}16(-) \\
16(-) \\
16(-)\end{array}$ & $\begin{array}{l}(-) \\
(-)\end{array}$ & $\begin{array}{l}(-) \\
( \pm) \\
(-)\end{array}$ & $\begin{array}{l}(+) \\
( \pm) \\
( \pm)\end{array}$ & $\begin{array}{l}0.88 \\
1.24 \\
1.10\end{array}$ & $\begin{array}{l}\text { Be } \\
\text { \#̈ } \\
\text { Decrease of poison } \\
\text { not evident }\end{array}$ \\
\hline $\begin{array}{c}2 \\
\text { H.0. } \\
27\end{array}$ & Dyspepsia & $\begin{array}{rc}\text { 5. } & \mathrm{XI} \\
9 . & , 1 \\
15 . & ",\end{array}$ & $\begin{array}{c}14(-) \\
1( \pm) \\
1(+)\end{array}$ & $\begin{array}{r}14(-) \\
1( \pm) \\
1(H)\end{array}$ & $\begin{array}{l}(-) \\
(-) \\
(-)\end{array}$ & $\begin{array}{l}(-) \\
(-)\end{array}$ & $\begin{array}{l}(-) \\
(-)\end{array}$ & $\begin{array}{l}0.62 \\
0.72 \\
0.36\end{array}$ & $\begin{array}{l}\text { Decrease of poison } \\
\text { evident }\end{array}$ \\
\hline $\begin{array}{c}3 \\
S . K \\
25\end{array}$ & Atrophy & $\begin{array}{ll}\text { 15. } & \mathrm{XI} \\
18 . & " \\
21 . & " \\
29 . & " n \\
8 . & \mathrm{XII}\end{array}$ & $\begin{array}{l}15(-) \\
16(-) \\
16(-) \\
16(-) \\
4(+)\end{array}$ & $\begin{array}{l}15(-) \\
14(-) \\
16(-) \\
16(-) \\
11( \pm)\end{array}$ & $\begin{array}{l}( \pm) \\
( \pm) \\
(-) \\
(-)\end{array}$ & $\begin{array}{l}(+) \\
(+) \\
( \pm) \\
(-) \\
(-)\end{array}$ & $\begin{array}{l}(+) \\
(+) \\
(+) \\
(-) \\
(-)\end{array}$ & $\begin{array}{l}1.75 \\
1.54 \\
0.88 \\
0.45 \\
0.56\end{array}$ & $\begin{array}{l}\text { Decrease of poison } \\
\text { evident }\end{array}$ \\
\hline $\begin{array}{c}4 \\
\mathrm{H} . \mathrm{Y} \\
24\end{array}$ & Dystrophy & $\begin{array}{l}\text { 14. I } \\
17 ., " \\
19 . ", \\
21 . ", \\
24 . ",\end{array}$ & $\begin{array}{l}15(-) \\
16(-) \\
16(-) \\
16(-) \\
4(-)\end{array}$ & $\begin{array}{l}15(-) \\
16(-) \\
16(-) \\
16(-) \\
10(-)\end{array}$ & $\begin{array}{l}(-) \\
(-) \\
(-) \\
(-)\end{array}$ & $\begin{array}{l}( \pm) \\
(-) \\
( \pm) \\
(-) \\
(-)\end{array}$ & $\begin{array}{l}(+) \\
(+) \\
( \pm) \\
(-) \\
(-)\end{array}$ & $\begin{array}{l}1.45 \\
1.10 \\
0.82 \\
0.88 \\
0.66\end{array}$ & $\begin{array}{l}\text { Decrease of poison } \\
\text { evident }\end{array}$ \\
\hline $\begin{array}{c}5 \\
\text { T.A. } \\
26\end{array}$ & Dystrophy & $\begin{array}{l}15 \text { I } \\
18 ", \\
22 ", \\
25 ",\end{array}$ & $\begin{array}{l}8(-) \\
14(-) \\
14(-) \\
11(-)\end{array}$ & $\begin{array}{l}14(-) \\
14(-) \\
16(-) \\
16(-)\end{array}$ & $\begin{array}{l}(-) \\
( \pm) \\
(-)\end{array}$ & $\begin{array}{l}(-) \\
(-) \\
(-)\end{array}$ & $\begin{array}{l}(-) \\
(-) \\
(-)\end{array}$ & \begin{tabular}{c|}
0 \\
0 \\
0.32 \\
0
\end{tabular} & $*$ \\
\hline $\begin{array}{c}6 \\
\text { T.M. } \\
36\end{array}$ & $\begin{array}{l}\text { Rhino- } \\
\text { pharyngi- } \\
\text { tis }\end{array}$ & $\begin{array}{l}\text { 15. I } \\
20 . \text { " } \\
22 . "\end{array}$ & $\begin{array}{r}5(-) \\
16(-) \\
16(-)\end{array}$ & $\begin{array}{l}16(-) \\
16(-) \\
16(-)\end{array}$ & $\begin{array}{l}( \pm) \\
( \pm) \\
( \pm)\end{array}$ & $\begin{array}{l}(-) \\
(-) \\
(-)\end{array}$ & $\begin{array}{l}( \pm) \\
(-) \\
(-)\end{array}$ & $\begin{array}{c}0.32 \\
0 \\
0\end{array}$ & $\begin{array}{l}* \\
\text { Decrease of poison }\end{array}$ \\
\hline $\begin{array}{c}7 \\
\text { M.A. } \\
27\end{array}$ & Bronchitis & $\begin{array}{l}\text { 10. I } \\
14 . " \\
18 . "\end{array}$ & $\begin{array}{r}12(-) \\
8(-) \\
16(-)\end{array}$ & $\left\{\begin{array}{l}15(-) \\
12(-) \\
16(-)\end{array}\right.$ & $\begin{array}{l}(-) \\
(-)\end{array}$ & $\begin{array}{l}(-) \\
(-)\end{array}$ & $\begin{array}{l}(-) \\
(-)\end{array}$ & $\begin{array}{c}0 \\
0.32 \\
0\end{array}$ & $\ddot{*}$ \\
\hline $\begin{array}{c}8 \\
\mathbf{K} . \mathbf{Y} . \\
\mathbf{3 0}\end{array}$ & Bronchitis & $\begin{array}{l}\text { 16. I } \\
20 ., " \\
23 . ",\end{array}$ & $\begin{array}{l}12(-) \\
16(-) \\
16(-)\end{array}$ & $\left\{\begin{array}{l}11(-) \\
14(-) \\
14(-)\end{array}\right.$ & $\left\{\begin{array}{l}( \pm) \\
(-) \\
(-)\end{array}\right.$ & $(-)$ & $\begin{array}{l}(-) \\
(-)\end{array}$ & $\begin{array}{l}0.36 \\
0.34 \\
0.38\end{array}$ & $\because$ \\
\hline $\begin{array}{c}9 \\
\text { T.I. } \\
25\end{array}$ & Bronchitis & $\begin{array}{l}\text { 17. I } \\
21 . ", \\
24 . "\end{array}$ & $\begin{array}{l}4( \pm) \\
1(H) \\
7( \pm)\end{array}$ & $\left\{\begin{array}{c}1(-) \\
7(-) \\
11( \pm)\end{array}\right.$ & $\begin{array}{l}(-) \\
(-)\end{array}$ & $\begin{array}{l}(-) \\
(-)\end{array}$ & $\begin{array}{l}(-) \\
(-)\end{array}$ & $\begin{array}{l}0.32 \\
0.44 \\
0.26\end{array}$ & $\begin{array}{l}* \\
* 4 \%\end{array}$ \\
\hline
\end{tabular}


TABle 7. Continued.

\begin{tabular}{|c|c|c|c|c|c|c|c|c|c|}
\hline \multirow{2}{*}{ 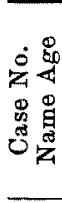 } & \multirow{2}{*}{$\begin{array}{c}\text { Diagnosis } \\
\text { of } \\
\text { infants }\end{array}$} & \multirow{2}{*}{$\begin{array}{c}\text { Date of } \\
\text { experi- } \\
\text { ment }\end{array}$} & \multicolumn{2}{|c|}{$\begin{array}{c}\text { Arakawa's } \\
\text { reaction }\end{array}$} & \multirow{2}{*}{ 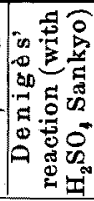 } & \multirow{2}{*}{ 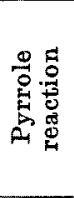 } & \multirow{2}{*}{ 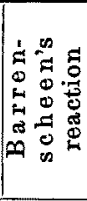 } & \multirow{2}{*}{ 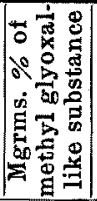 } & \multirow{2}{*}{ Remarks } \\
\hline & & & Right & Left & & & & & \\
\hline $\begin{array}{c}10 \\
\text { I. K. } \\
26\end{array}$ & Atrophy & $\begin{array}{l}\text { 27. I } \\
29 . \text { " } \\
\text { 1. II } \\
\text { 5. " }\end{array}$ & $\begin{array}{c}16(-) \\
5( \pm) \\
8(-) \\
8(-)\end{array}$ & $\begin{array}{c}16(-) \\
15(-) \\
8(-) \\
12(-)\end{array}$ & $\begin{array}{l}(-) \\
(-) \\
(-) \\
(-)\end{array}$ & $\begin{array}{l}(-) \\
(-) \\
(-)\end{array}$ & $\begin{array}{l}(-) \\
(-) \\
(-)\end{array}$ & $\begin{array}{l}0.76 \\
1.10 \\
0.66 \\
0.36\end{array}$ & $\begin{array}{l}\text { Decrease of poison } \\
\text { evident }\end{array}$ \\
\hline $\begin{array}{c}11 \\
\text { I. M. } \\
23\end{array}$ & $\begin{array}{l}\text { Pneumo- } \\
\text { nia }\end{array}$ & $\begin{array}{l}\text { 28. I } \\
\text { 30. " } \\
\text { 31. " }\end{array}$ & $\begin{array}{l}16(-) \\
16(-) \\
16(-)\end{array}$ & $\begin{array}{l}16(-) \\
16(-) \\
16(-)\end{array}$ & $\begin{array}{l}(-) \\
(-)\end{array}$ & $\begin{array}{l}(-) \\
(-)\end{array}$ & $\begin{array}{l}(-) \\
(-)\end{array}$ & $\begin{array}{l}0.34 \\
0.35 \\
0.35\end{array}$ & $*$ \\
\hline $\begin{array}{c}12 \\
\text { T. T. } \\
26\end{array}$ & Atrophy & $\begin{array}{r}\text { 1. II } \\
4 . ", \\
7 . " \\
15 . "\end{array}$ & $\begin{array}{r}6( \pm) \\
16(-) \\
2(-) \\
2(-)\end{array}$ & $\begin{array}{c}10(-) \\
3(-) \\
5(-) \\
2(-)\end{array}$ & $\begin{array}{l}(-) \\
(-) \\
(-)\end{array}$ & $\begin{array}{l}(-) \\
(-) \\
(-)\end{array}$ & $\begin{array}{l}(-) \\
(-) \\
(-)\end{array}$ & $\begin{array}{c}0 \\
0 \\
0.35 \\
0\end{array}$ & $*$ \\
\hline $\begin{array}{r}13 \\
\text { S.I. } \\
25\end{array}$ & $\begin{array}{l}\text { Pneumo- } \\
\text { nia }\end{array}$ & $\begin{array}{l}\text { 12. II } \\
16 . ", \\
20 . "\end{array}$ & $\begin{array}{r}15(-) \\
7(-) \\
14(-)\end{array}$ & $\begin{array}{r}11(-) \\
7(-) \\
2(-)\end{array}$ & $\begin{array}{l}(-) \\
(-)\end{array}$ & $\begin{array}{l}(-) \\
(-)\end{array}$ & $\begin{array}{l}(-) \\
(-)\end{array}$ & $\begin{array}{c}0 \\
0.32 \\
0\end{array}$ & $\%$ \\
\hline $\begin{array}{c}14 \\
\text { S. A. } \\
26\end{array}$ & Bronchitis & $\begin{array}{l}\text { 18. II } \\
25 . " n \\
28 . \text { " } \\
\text { 1. III } \\
\text { 4. " }\end{array}$ & $\begin{array}{l}6(-) \\
2( \pm) \\
1( \pm) \\
1( \pm) \\
4(-)\end{array}$ & $\begin{array}{r}3(-) \\
3( \pm) \\
1( \pm) \\
4( \pm) \\
16(-)\end{array}$ & $\begin{array}{l}(-) \\
(-) \\
(-) \\
(-)\end{array}$ & $\begin{array}{l}(-) \\
(-) \\
(-) \\
(-)\end{array}$ & $\begin{array}{l}(-) \\
(-) \\
(-) \\
(-)\end{array}$ & $\begin{array}{c}0.32 \\
0 \\
0 \\
0.35 \\
0\end{array}$ & * \\
\hline $\begin{array}{r}15 \\
\text { K.N. } \\
27\end{array}$ & $\begin{array}{l}\text { Pneumo- } \\
\text { nia }\end{array}$ & 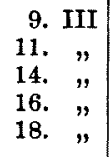 & $\begin{array}{r}12(-) \\
6(-) \\
13(-) \\
11( \pm) \\
11(-)\end{array}$ & $\begin{array}{c}6(-) \\
15(-) \\
13( \pm) \\
3( \pm) \\
6(-)\end{array}$ & $\begin{array}{l}(-) \\
(-) \\
(-) \\
(-)\end{array}$ & $\begin{array}{l}(-) \\
( \pm) \\
( \pm) \\
( \pm)\end{array}$ & $\begin{array}{l}( \pm) \\
(-) \\
(-)\end{array}$ & $\begin{array}{c}0 \\
0.32 \\
0.36 \\
0 \\
0.34\end{array}$ & 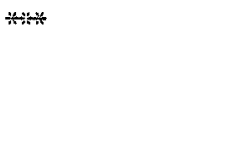 \\
\hline \begin{tabular}{c|}
16 \\
Y.H. \\
24
\end{tabular} & Bronchitis & $\begin{aligned} 25 . & \text { IV } \\
7 . & V \\
11 . & " y\end{aligned}$ & $\begin{array}{l}1(+) \\
1(H) \\
1(H)\end{array}$ & $\begin{array}{l}1(+) \\
1(H) \\
1(H)\end{array}$ & $\begin{array}{l}(-) \\
(-)\end{array}$ & $\begin{array}{l}(-) \\
(-)\end{array}$ & $\begin{array}{l}( \pm) \\
(-)\end{array}$ & $\begin{array}{c}0.75 \\
0.36 \\
0\end{array}$ & $\begin{array}{l}\text { Decrease of poison } \\
\text { evident }\end{array}$ \\
\hline
\end{tabular}

Be Mother is suffering from beriberi.

* Has been taking vitamin-B.

** Yakriton (1/2 Unit) injected every other day.

柴* No treatment.

reaction, pyrrole reaction and Deni gè s' reaction-for methyl glyoxal; and further between these qualitative reactions on one side and $\mathrm{F}$ is $\mathrm{ch}$ ler and Boethner's ${ }^{17}$ quantitative method on the other. But if 
lactating mothers were grouped into three groups; the strongly or normally Arakawa-positive, and the weakly Arakawa-positive* and the completely Arakawa-negative** groups, then a more or less close relation between the qualitative reactions and the quantitative method will be seen. In the strongly or normally Arakawa-positive group the qualitative reactions were negative in almost all the cases and the poison content was also small generally.

In the weakly Arakawa-positive and the completely Arakawanegative group, the qualitative reactions were positive in the majority of cases and the poison content was also larger in these groups. The difference between the latter and the former two groups might have been more striking, if those mothers who had been taking vitamin-B preparations were examined before they began to take them. It will thus be seen from the content of the urine in the methyl glyoxal-like substance that the former two groups were far more in a state of avitaminosis- $B$ than the latter group. And the fact will further be made more evident, if the effect of vitamin-B administration upon Arakawanegative mothers is seen.

\section{Conclusions.}

As has been repeatedly shown from this Laboratory, Arakawanegative mothers are in a state or on the verge of B-avitaminosis. Though there is no strict coincidence among the three qualitative tests -Barrenscheen's reaction, pyrrole reaction and Denigès' reaction-for methyl glyoxal, and again between these tests on one side and Fischler and Boethne r's ${ }^{17}$ quantitative method on the other, these tests'are more often positive in the Arakawa-negative group of mothers than in the Arakawa-positive, and the quantitative method shows a poison content to be much larger in the former group than in the latter. Besides, an administration of vitamin-B to Arakawa-negative mothers will make the poison content in the urine smaller.

\footnotetext{
**
} 